\title{
OBJECT-ORIENTED CHANGE DETECTION BASED ON MULTI-SCALE APPROACH
}

\author{
Yonghong Jia ${ }^{\text {a }}$, Mingting Zhou ${ }^{\text {a }}$, Ye Jinshan ${ }^{\mathrm{b}}$ \\ ${ }^{a}$ Institute of Remote Sensing and Information Engineering, Wuhan University, Wuhan 430079, China-(469630944, \\ 1849101245)@qq.com \\ b Institute of Remote Sensing and Digital Earth, Beijing 100101, China-yejs@irsa.ac.cn
}

Commission VII, WG VII/5

KEY WORDS: Optimal scale; multi-scale; CART tree; object-oriented techniques

\begin{abstract}
:
The change detection of remote sensing images means analysing the change information quantitatively and recognizing the change types of the surface coverage data in different time phases. With the appearance of high resolution remote sensing image, objectoriented change detection method arises at this historic moment. In this paper, we research multi-scale approach for high resolution images, which includes multi-scale segmentation, multi-scale feature selection and multi-scale classification. Experimental results show that this method has a stronger advantage than the traditional single-scale method of high resolution remote sensing image change detection.
\end{abstract}

\section{INTRODUCTION}

Change detection is the process of identifying differences observing at different times (Bruzzone L., 2000). In the past decades, remotely sensed images have become a major data source for different applications of change detection.

A wide variety of change detection methods have been developed. Some of the most commonly used traditional change detection techniques are image differencing, principal component analysis, post-classification comparison and change vector analysis. These techniques have been typically applied and evaluated in medium spatial resolution satellite images such as Landsat TM.

However, when it comes to the study of change detection for high resolution images, methods mentioned above have some drawbacks, for the reason that the traditional pixel-based change detection methods are built on the assumption that neighbouring pixels are relatively independent to each other while in high resolution images, several adjacent pixels combine together to make up a significant geographical object. A wide variety of experiments have shown that for images with a resolution higher than 10 meters, object-oriented change detection methods perform better than the traditional ones. On the other hand, traditional remote sensing image change detection methods built on the pixel level are mainly based on the analysis of the spectral information and hardly analyse the shape features and structure features of ground objects. However, high resolution remote sensing image has brought significant changes to the remote sensing technology. It can very clearly show the structure, texture and detail information of the landscape. In addition to getting the spectral features, object-based methods can also get the structure, shape and texture information of the surface objects, making it easier to solve problems in high resolution remote sensing image change detection. In general, it is of great significance to study on object-based change detection techniques for high resolution images.

There have been many object-oriented change detection methods for high resolution images (Listner C., Niemeyer I., 2011). The most basic ideas of object-oriented approach is to segment the image and regard the objects as the basic unit of operation. Most of the existed object-oriented change detection methods tend to segment the image in single scale. However, in high resolution images, the scales of different land-cover types are different; objects of the same type also have different scales. Single scale segmentation cannot adequately reflect the characteristics of different objects presented in images.

In this paper, an innovative object-oriented change detection method is proposed. Firstly, the image is segmented in several optimal segmentation scales. Then object features including spectral features, texture features and structure features are extracted and evaluated in different scales to serve for the next step. Thirdly, a novel multi-scale classifier based on decision tree is put forward to obtain the class information of each object. The last step is to compare the class information of different time phases to figure out whether change exists. Due to the good use of main characters of object-oriented thinking, this novel method can give full play to the characteristics of high resolution images and get much better result than traditional methods for detecting changes from high resolution remote sensing images.

\section{METHODOLOGY}

\subsection{Multi-scale Segmentation and Optimal Segmentation} Scales Selection

The first step of the object-oriented change detection process is to segment the image into objects. Segmentation scale is of great

* Corresponding author: Mingting Zhou, Department of Remote Sensing and Information Engineering, WuHan University, Hubei Province, China. Phone 15994261867, E-mail: 1849201245@qq.com 
importance for segmentation, which directly determines the size of objects as well as the accuracy of information extraction ( $\mathrm{Su}$ X.Q. et al, 2011). Different kinds of ground objects have their own optimal segmentation scales. For example, the best scale for buildings is obviously smaller than that for forest and grassland. Different segmentation scales can generate polygons with great difference. The smaller the scale is, the more the polygons are generated and the smaller the size of a single polygon is (Woodcock, Curtis E., Alan H., 1987). Based on the above analysis, this article intends to maximize the change detection accuracy by making a fusion of the change detection results in various optimal segmentation scales.

The best segmentation scale for a certain ground object should satisfy the following conditions:

- Objects segmented from its optimal segmentation scale can maintain the inherent spatial structure features of the target object;

- After segmentation, the object must be purified, that is, only one class can exist in one object.

Taking calculation efficiency into account, the optimal scale should be the biggest scale that satisfies conditions mentioned above. In order to find the optimal scale, this article pays attention to the change trend of local variance (LV) of segmented objects with scales. With the segmentation scale growing bigger, if there is an abrupt change of the LV, the corresponding scale is considered as the optimal one.

The steps of choosing optimal segmentation scales in this paper are as following:

- Clip a small piece of image as sample image. In the sample image, concerning ground objects should be included entirely.

- Determine the range of interested scales and segment the sample image into multiple scales.

- Calculate LV of objects segmented with multiple scales.

- Do regression analysis to get a LV-Scale curve with $\mathrm{LV}$ as the $\mathrm{y}$-axis and scale as the $\mathrm{x}$-axis.

- Take a derivative of the LV-Scale curve to obtain the ROC curve. Scales corresponding to the ROC curve's peaks are considered as the optimal scales.

Because of the complexity of surface features, there may be not only one peak in the ROC curve. We can add some subjective analysis and choose one peak as the optimal scale for each investigated class according to the real situation.

In this paper we use eCognition software to perform a multiscale segmentation. Besides segmentation scale, spectral and shape heterogeneity weights and smoothness and compactness weights are also very important parameters in the process of multi-scale segmentation (Zhang JP., et al, 2011.). In this article, these parameters are decided mainly by the expert experience.

\subsection{Optimal Features of Objects Selection}

After image segmentation, eCognition can obtain objects features by evaluating image objects themselves as well as their embedding in the image objects hierarchy. There are many objects features that can be categorized into five classes including spectral features, texture features, shape features, hierarchy features and thematic attributes (Smits, Paul C., Alessandro A., 1999).

In this paper, we only use spectral features, texture features and shape features. Not all features are suitable to get the best change detection result. How to choose the most suitable features for change detection is an interest of many researchers. There have been many feature selection algorithms, among which the Fisher Score is the most classic one.

Fisher Score is supervised with class labels, by seeking features with best discriminant ability (Xu, H.Y., 2012). The Fisher Score of the $\mathrm{r}^{\text {th }}$ feature, which should be maximized, is computed by formula (1):

$$
F_{r}=\frac{\sum_{w=1}^{c} n_{w}\left(\mu_{w r}-\mu_{r}\right)^{2}}{\sum_{w=1}^{c} n_{w} \delta_{\mathrm{wr}}^{2}}
$$

where $\mathrm{n}_{\mathrm{w}}=$ the number of samples in class $\mathrm{w}$

$$
\begin{aligned}
& \mu_{w r}=\text { the mean of class } \mathrm{w} \\
& \delta_{\mathrm{wr}}{ }^{2}=\text { the variance of class } \mathrm{w} \\
& \mathrm{w}=1, \ldots, \mathrm{c} .
\end{aligned}
$$

According to Fisher Score, a ranked features list can be effectively obtained, hence we can remove relatively unimportant features or redundant features.

Obviously, different types of features have different performance in different scales. For example, spectral features are more concentrated in small scales, while in large scales, objects are segmented with integrity so structure features will be presented better. It makes sense to take feature's scale property into consideration when select features. This paper revises the traditional Fisher Score method based on the above analysis. Steps of the improved feature selecting method are as followed:

- Multi-scale sample selection. Select samples from the multi-scale segmented object levels. Export spectral features in the small scale level, texture features in the midscale level and structure features in the big scale level.

- Calculate Fisher Scores. Separately calculate the Fisher Scores of features exported from different object levels and sort the results in order.

- Features selection. Features with the highest scores in different levels are the most effective features for change detection.

\subsection{Multi-scale CART Tree Classification}

Decision tree is a machine mining learning method, which has the characteristics of non-parametric, and is able to handle noisy data and select the characteristics automatic, contributing great advantages to remote sensing classification. Mature tree construction methods include ID3, C4.5, C5.0 series, CART, SLIQ, SPRINT and CHAID (Ludwig, S., Jakobovic D., Picek, 2015). Among these mature methods, CART tree is a relatively stable one for classification.

As is stated above, different kinds of ground objects have their own optimal segmentation scales. In order to adapt to the scale characters of different ground objects, this article takes the CART tree to extract different classes on their own optimal segmentation scales individually. The classification results in different levels will be aggregated together on the smallest segmentation level. 
Compared with traditional CART tree classification methods, multi-scale CART tree classification method takes the scale characteristics of ground objects into consideration and can get a better classification result.

\subsection{Post Classification Comparison Change Detection}

Post-classification change detection is one of the most common methods used for change detection. It is very advantageous when using data from different sensors with different spatial and spectral resolutions. Compared with other change detection methods, post-classification method can figure out the information about types of changes that have occurred. But it is important to note that this method depends on the results of the classification of both images.

\section{EXPERIMENTS AND RESULTS ANALYSIS}

\subsection{Test Data Description}

For this study, two data sets of multiple temporal high resolution imagery, which consists of four multispectral bands, were acquired. The data specifications are described in Table 1 .

Table 1 Data Specification

\begin{tabular}{|c|c|c|c|c|}
\hline & \multicolumn{2}{|c|}{ Case 1} & \multicolumn{2}{|c|}{ Case 2} \\
\hline Sensor & $\begin{array}{l}\text { World } \\
\text { View-I }\end{array}$ & Pleiades & $\begin{array}{l}\text { World } \\
\text { View-I }\end{array}$ & Pleiades \\
\hline Location & \multicolumn{2}{|c|}{ Wuhan Luoli village } & \multicolumn{2}{|c|}{ Wuhan Lujiazhuang } \\
\hline Date & 2012.8 & 2013.11 & 2012.8 & 2013.11 \\
\hline $\begin{array}{l}\text { Spectral } \\
\text { resolution }\end{array}$ & \multicolumn{4}{|c|}{ Panchromatic and four multi-spectral bands } \\
\hline $\begin{array}{c}\text { Spatial } \\
\text { resolution }\end{array}$ & \multicolumn{4}{|c|}{0.5 meter } \\
\hline Image size & \multicolumn{4}{|c|}{$4000 * 4000$ pixels } \\
\hline
\end{tabular}

The standard false color images of two cases are showed in Figure 1.

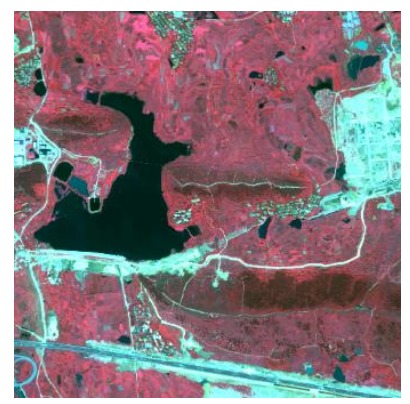

Case 1: Year 2012

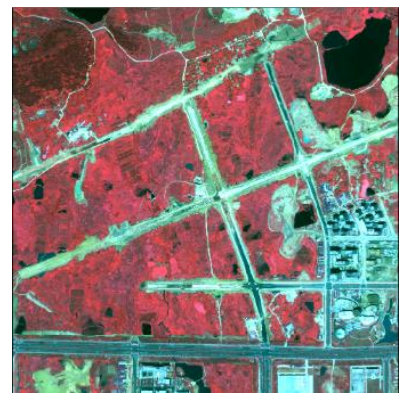

Case 2: Year 2012

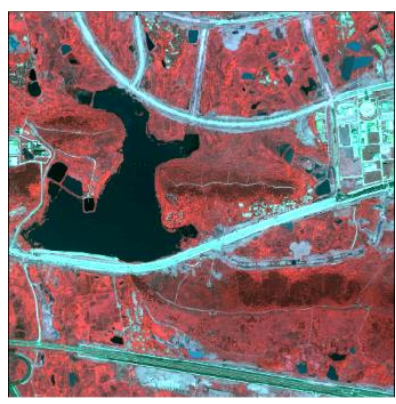

Case 1: Year 2013

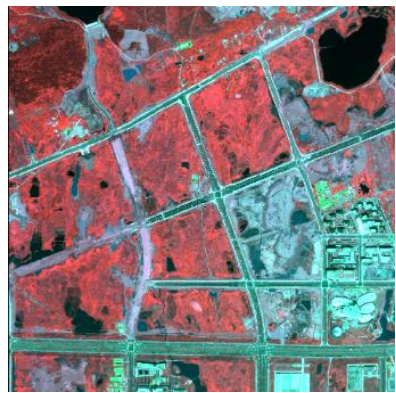

Case 2: Year 2013
Figure 1 Test Data
The study images include both complex and simple changes of the man-made construction. This data represents a typical changing form of urban development, including changing types between plant, water and build-ups. The image pre-processing includes geometric correction, image registration and histogram matching referenced by the second image.

\subsection{Multi-scale Segmentation and Optimal Segmentation Scales Selection}

It is of essential importance to choose optimal segmentation scales before segmentation. Steps to choose the optimized scale have been presented in Section 2.1. Trends that LV and ROC change with segmentation scales are showed in Figure 2.

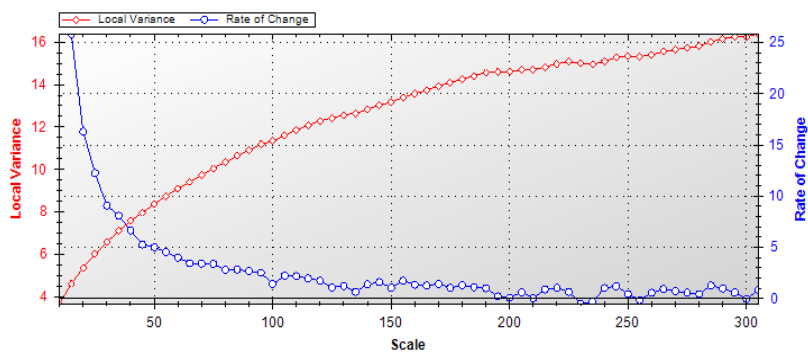

Figure $2 \mathrm{LV}$ and ROC Curves

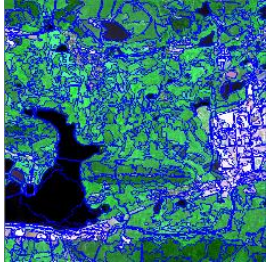

Scale $=285$

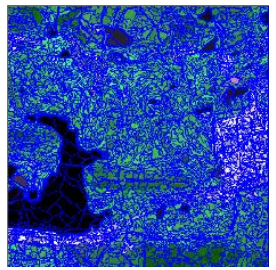

Scale $=105$

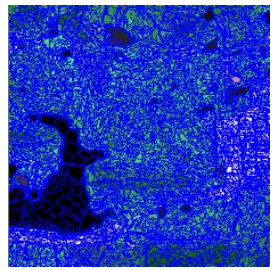

Scale $=75$
Figure 3 Segmentation Results

It can be found in Figure 2 that the peaks of ROC curve appears at scale $75,105,130,145,155,170,180,220,246,265,285$. We choose three typical scales to extract our three targeted ground objects, while scale 75 for build-ups, 105 for plants and 285 for water. Figure 3 shows the multi-scale segmentation results in the three optimal scales.

\subsection{Optimal Features of Objects Selection}

After image segmentation, eCognition can obtain objects features by evaluating image objects themselves as well as their embedding in the image object hierarchy.

In this paper, different kinds of features are evaluated in different segmentation levels using the novel feature selection method proposed in Section 2.2. In the process of feature selection, features including mean features, HIS features, index features like NDVI and NDWI and other spectral features are estimated in small scale 75; texture features like contrast, compactness, entropy, homogeneity and density are evaluated in medium scale 105; and structure features including length/width, length, shape index, asymmetry, area and border length are all calculated in big scale 285 .

Table 2 is the fisher scores of features extracted from different levels. 
Table 2 Fisher Scores of Multi-scale Features

\begin{tabular}{|c|c|c|c|}
\hline \multicolumn{4}{|c|}{ Scale=285 Structure Features } \\
\hline Feature & $\begin{array}{c}\text { Fisher } \\
\text { Score }\end{array}$ & Feature & $\begin{array}{c}\text { Fisher } \\
\text { Score }\end{array}$ \\
\hline Length/Width & 0.3502 & Asymmetry & 0.0770 \\
\hline Length & 0.2738 & Area & 0.0657 \\
\hline Shape Index & 0.0900 & Border Length & 0.0281 \\
\hline \multicolumn{4}{|c|}{ Scale=105 Texture Features } \\
\hline Feature & $\begin{array}{c}\text { Fisher } \\
\text { Score }\end{array}$ & Feature & $\begin{array}{c}\text { Fisher } \\
\text { Score }\end{array}$ \\
\hline Contrast & 1.6711 & Homogeneity & 0.0709 \\
\hline Compactness & 0.2048 & Density & 0.0664 \\
\hline Entropy & 0.2047 & & \\
\hline \multicolumn{4}{|c|}{ Scale=75 Spectral Features } \\
\hline Feature & $\begin{array}{c}\text { Fisher } \\
\text { Score }\end{array}$ & Feature & $\begin{array}{c}\text { Fisher } \\
\text { Score }\end{array}$ \\
\hline Max_diff & 4.8490 & Brightness & 0.1143 \\
\hline Intensity & 0.8742 & Blue & 0.0976 \\
\hline Mean_red & 0.7839 & STD_Blue & 0.0654 \\
\hline Mean_green & 0.5667 & STD_Green & 0.0619 \\
\hline Mean_NIR & 0.4416 & STD_NIR & 0.0418 \\
\hline Hue & 0.4175 & Saturation & 0.0107 \\
\hline NDVI & 0.2676 & STD_Red & 0.0001 \\
\hline NDWI & 0.1914 & & \\
\hline
\end{tabular}

We can instantly analyse from Table 2 that structure features like Length/Width and Length, texture features like Contrast, Compactness and Entropy, spectral features like Max_diff, Intensity, Mean Features, Hue, NDVI, NDWI and Brightness, all of these features get high Fisher Scores in the evaluating process. It means that features stated above plays a more important role in classification than those abandoned features.

\subsection{Single-scale CART Tree Classification}

In order to convince that the idea to classify different kind of objects in their corresponding optimal segmentation scales can improve the quality of classification, a comparative experiment to conduct classification in single-scale is firstly done. In the contrast experiment, all the classes including plant, water and build-up are classified on the scale 75 using CART tree. Features input into the single-scale CART tree are all features selected in Section 3.3. Figure 4 shows the results of single-scale CART tree classification.

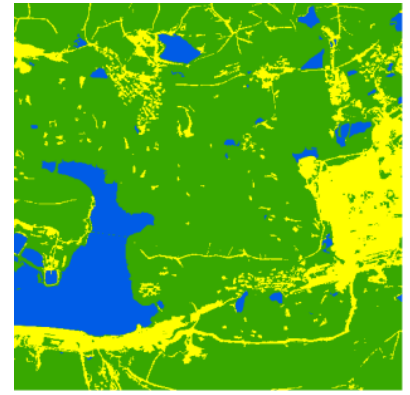

(a) Case 12012

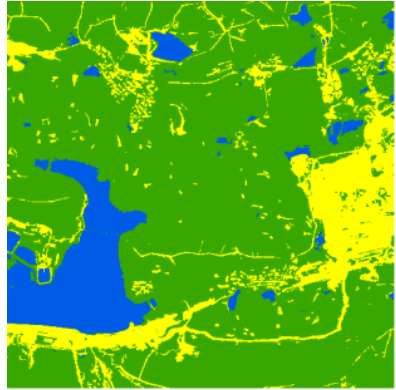

(b) Case 12013

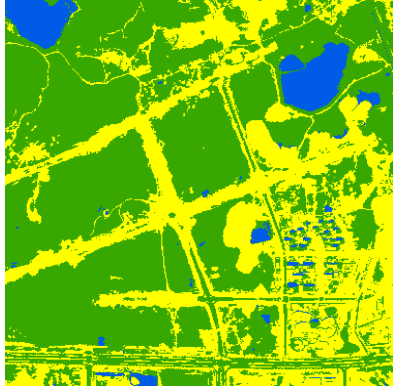

(c) Case 22012 Legend

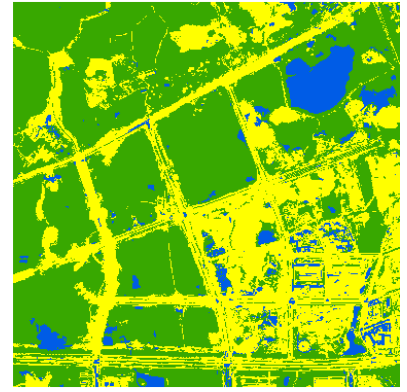

(d) Case 22012

$$
\text { buildup plant } \square \text { water }
$$

Figure 4 Classification Results of Single-scale CART Tree

The classification result of single-scale CART tree is a bit of terrible visually, while many objects are wrongly classified and the confusion between three classes is very serious. Table 3 gives the quantitative evaluation of the results of single-scale CART tree classification.

Table 3 Accuracy of Single-scale CART Tree

\begin{tabular}{|c|c|c|c|c|}
\hline Class & Data & $\begin{array}{c}\text { Product } \\
\text { Accuracy }\end{array}$ & $\begin{array}{c}\text { User } \\
\text { Accuracy }\end{array}$ & $\begin{array}{c}\text { Overall } \\
\text { Accuracy }\end{array}$ \\
\hline \multirow{4}{*}{ plant } & \multirow{2}{*}{ Case 1} & 0.822386 & 0.95855 & \multirow{12}{*}{0.78} \\
\hline & & 0.827677 & 0.991113 & \\
\hline & \multirow{2}{*}{ Case 2} & 0.540260 & 0.995258 & \\
\hline & & 0.672468 & 0.953445 & \\
\hline \multirow{4}{*}{ water } & \multirow{2}{*}{ Case 1} & 0.847291 & 0.934783 & \\
\hline & & 0.646697 & 0.886316 & \\
\hline & \multirow{2}{*}{ Case 2} & 0.775316 & 0.218166 & \\
\hline & & 0.448649 & 0.922222 & \\
\hline \multirow{4}{*}{ build-up } & \multirow{2}{*}{ Case 1} & 0.922425 & 0.699904 & \\
\hline & & 0.985372 & 0.757214 & \\
\hline & \multirow{2}{*}{ Case 2} & 0.995873 & 0.619914 & \\
\hline & & 0.964816 & 0.723165 & \\
\hline
\end{tabular}

\subsection{Multi-scale CART Tree Classification}

As analysed above, multi-scale classification is theoretically beneficial to improve classification effects. This part will confirm this assumption practically. Before classification, it is of great importance to build the object inheritance relationships among three levels, which is showed in Figure 5.

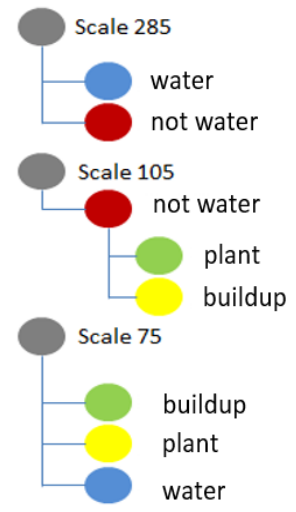

Figure 5 Object inheritance relationships 


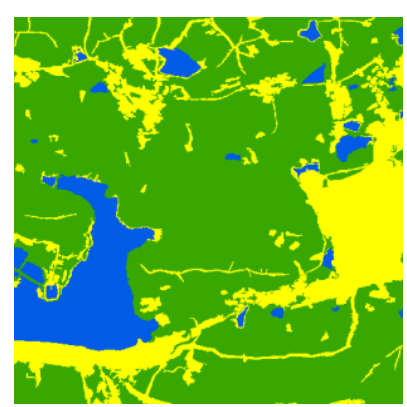

(a) Case 12012

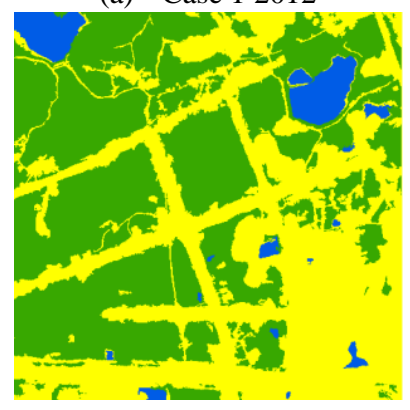

(c) Case 22012 Legend

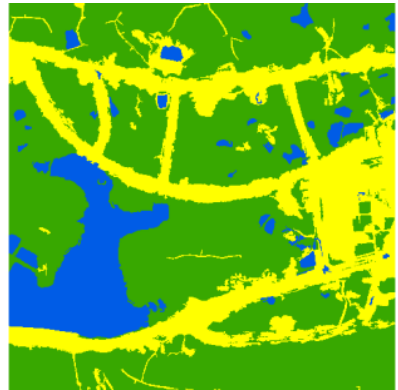

(b) Case 12013

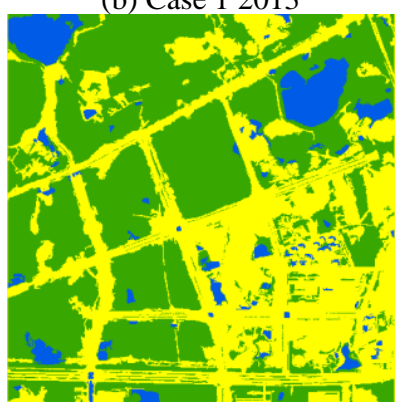

(d) Case 22013 buildup

plant

water

The results of multi-scale CART tree classification is presented in Figure 6.

By analyzing the above results, we can find that the results of multi-scale CART tree is better than the results of single-scale CART tree apparently. Boundaries of objects are much clearer and classes are less confused in the multi-scale classification method. It seems that the small scale does not equal to high accuracy. What's the most important is to find the optimal segmentation scale for each class and extract them according to their characteristics. Table 4 will manifest the not-confirmed conclusion stated before quantitatively.

Table 4 Accuracy of Multi-scale CART Tree

\begin{tabular}{|c|c|c|c|c|}
\hline Class & Data & $\begin{array}{l}\text { Product } \\
\text { Accuracy }\end{array}$ & $\begin{array}{c}\text { User } \\
\text { Accuracy }\end{array}$ & $\begin{array}{c}\text { Overall } \\
\text { Accuracy }\end{array}$ \\
\hline \multirow{4}{*}{ plant } & \multirow{2}{*}{ Case 1} & 0.950396 & 0.906655 & \multirow{12}{*}{0.86} \\
\hline & & 0.990463 & 0.820377 & \\
\hline & \multirow[b]{2}{*}{ Case 2} & 0.937616 & 0.863451 & \\
\hline & & 0.994862 & 0.537471 & \\
\hline \multirow{4}{*}{ water } & \multirow{2}{*}{ Case 1} & 0.972826 & 0.733606 & \\
\hline & & 0.797895 & 0.690346 & \\
\hline & \multirow{2}{*}{ Case 2} & 0.788889 & 0.529851 & \\
\hline & & 0.800633 & 0.314286 & \\
\hline \multirow{4}{*}{ build-up } & \multirow{2}{*}{ Case 1} & 0.836925 & 0.917489 & \\
\hline & & 0.757214 & 0.98374 & \\
\hline & \multirow{2}{*}{ Case 2} & 0.907633 & 0.960185 & \\
\hline & & 0.950396 & 0.906655 & \\
\hline
\end{tabular}

On the whole, the overall accuracy of multi-scale CART tree method has increased by $8 \%$ than that of the single-scale CART tree method.

\subsection{Post-Classification Comparison Change Detection}

Comparing the above two classification results, we can see the multi-scale classification results are more effective than the single-scale classification results. In this paper, we choose multiscale classification results to do change detection. The result of object-oriented change detection is showed in Figure 7.

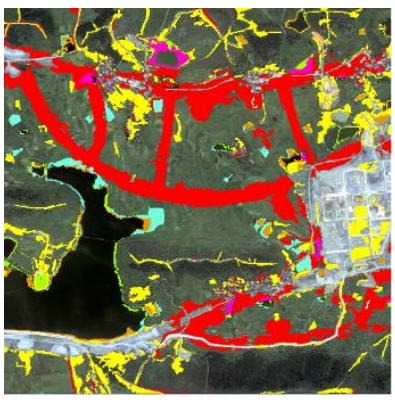
Legend

buildup to plant

buildup to water plant to water

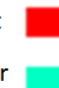

plant to buildup

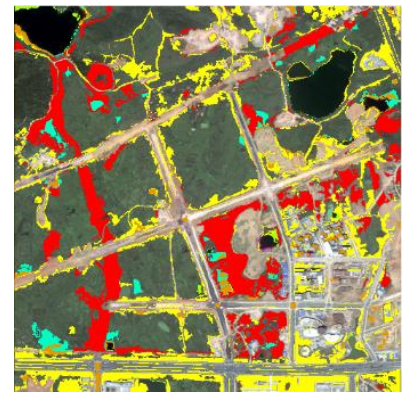

Case 2
Figure 7 Change Detection Results

Table 5 gives the accuracy of change detection result by the method proposed in this paper. The accuracy of three classes are as high as $90 \%$, while the false alarm and omission factor is lower than $20 \%$. These statistics confirm that multi-scale analysis is beneficial to improve the effects of object-based change detection.

Table 5 Accuracy of Change Detection

\begin{tabular}{|c|c|c|c|c|}
\hline \multirow{2}{*}{ Class } & Data & $\begin{array}{c}\text { Accuracy } \\
(\%)\end{array}$ & $\begin{array}{c}\text { False } \\
\text { alarm (\%) }\end{array}$ & $\begin{array}{c}\text { Omission } \\
\text { factor } \\
(\%)\end{array}$ \\
\hline \multirow{2}{*}{ plant } & Case 1 & 98.33 & 2.47 & 4.73 \\
\cline { 2 - 5 } & Case 2 & 90.91 & 1.46 & 2.72 \\
\hline \multirow{2}{*}{ water } & Case 1 & 95.65 & 12.77 & 6.55 \\
\cline { 2 - 5 } & Case 2 & 95.89 & 3.58 & 16.23 \\
\hline \multirow{2}{*}{ build-up } & Case 1 & 92.96 & 11.00 & 2.40 \\
\cline { 2 - 5 } & Case 2 & 92.53 & 19.98 & 5.21 \\
\hline
\end{tabular}

\section{CONCLUSIONS}

In this paper, we research multi-scale approach for high resolution images, which includes multi-scale segmentation, multi-scale feature selection and multi-scale classification. The "multi-scale" idea puts emphasis on the scale characteristics of different objects. Firstly, it is considered that different kinds of ground objects have different scale characteristics, so they should be segmented on their own optimal segmentation scales. Then, features are also regarded as scale-concerning, thus they are selected and evaluated in multi-scales like spectral features in small scale, texture features in medium scale and structure scales in big scale. Based on the above analysis, a new method of multi-scale CART tree classification is proposed, raising the classification accuracy by $8 \%$ than the traditional single-scale CART tree. Eventually, the results of change detection in this paper are satisfactory. The idea of multi-scale approach in object-oriented change detection for high resolution images is promising and better than those single-scale approaches. 


\section{ACKNOWLEDGEMENTS}

Work described in this paper was jointly supported by Road traffic in the field of civil military integration demonstration projects (GFZX0404080102).

\section{REFERANCES}

Bruzzone L., 2000. Prieto D F. Automatic analysis of the difference image for unsupervised change detection. IEEE Transactions on Geoscience and Remote Sensing, 38(3), pp. $1171-1182$.

Xu, H.Y., 2012. Face recognition based on Multi-class Fisher Scores. Fuzzy Systems and Knowledge Discovery (FSKD), 2012 9th International Conference on IEEE, pp.1221-1224.

Listner C., Niemeyer I., 2011. Recent advances in object-based change detection. Geoscience and Remote Sensing Symposium (IGARSS), IEEE International. IEEE, pp.110-113.

Ludwig, S., Jakobovic D., Picek, 2015. Analyzing Gene Expression Data: Fuzzy Decision Tree Algorithm applied to the Classification of Cancer Data. FUZZ-IEEE 2015

Smits, Paul C., Alessandro A., 1999. Updating land-cover maps by using texture information from very high-resolution spaceborne imagery. IEEE Transactions on Geoscience and Remote Sensing, 37(3), pp. 1244-1254.

Su XQ. et al, 2011. Land-Use and Land-Cover Change Detection Based on Object-Oriented Theory. Image and Data Fusion (ISIDF), International Symposium on IEEE, pp. 1-4.

Zhang JP., et al, 2011. Multi-scale segmentation in change detection for urban high resolution images. Geoscience and Remote Sensing Symposium (IGARSS), IEEE International IEEE, pp. 209-212.

Woodcock, Curtis E., Alan H., 1987. The factor of scale in remote sensing. Remote sensing of Environment, 21(3), pp. 311332 . 